\title{
Infertility Caused By the Early Failure of Ovaries Has Been Cured By TCM with A Natural Pregnancy
}

\author{
Ms Dan Jiang* \\ Hallam Institute of TCM, UK \\ *Corresponding author: Ms Dan Jiang, Hallam Institute of TCM, 439 Glossop Road Sheffield S10 2PR, UK.

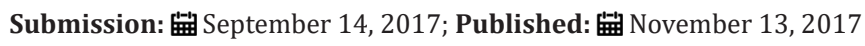

\section{Case Report}

The roles of Traditional Chinese Medicine (TCM) in Secondary Infertility Patients due to Ovary Failure and pregnancy Development among Females of Reproductive age in Sheffield.

\section{Introduction}

Traditional Chinese Medicine (TCM) plays as the main role to allow natural development of natural pregnancy, after the basic drug for secondary infertility with nonresponsive patients on the modern medicine such as oestrogen pills due to diseases such as ovary failure [1].

\section{Material}

Mrs J W, 29 years old, who is a medical researcher, has irregular menstruation for all of her life. She has to take oestrogen pills to make her regular period. After her marriage plans pregnancy, she has not had her period for more than two years after she stops her oestrogen pill. Her oestrogen level remained low $(:<50)$, this is menopause level. ) So she is diagnosed as an early failure of ovaries by Gynecologist. She at initial visits me only for making her relax with acupuncture. Due to prolonged untrust on traditional Chinese herbal medicine, she refused taking them. But after she feels progressed with acupuncture, then her taking some patent herbal medicines, until her taking full herbal medicine prescription. Following an 11 months of TCM treatment, she has built her menstrual circles, and become a natural conceive. After laboured a healthy baby girl, she returns her regular menstrual circles and have another natural pregnancy in two years without any support. So I believe that the early failure of ovaries had been cured for her [2].

\section{Clinical figures when she visit me at initial}

Depression, insomnia, nervous, restlessness etc. pale complexion, light tongue with thin white coating and weak pulse.

\section{Treating principle}

Due to being a medical researcher, she initially has not enough confident to TCM. After trying acupuncture weekly at first, then

patent herbs being added for three months, she finds that her oestrogen level increases to 65 . So she expects to try the best way in TCM. I change her from patent herbal form to dry herbal prescription and keep her regular acupuncture weekly. The pattern of TCM differentiation is Kidney Yang deficiency with Qi stagnation and blood stasis, to strengthen Qi and warm Yang, to remove Qi stagnation and blood stasis as the treating principle of TCM (Figure 1).

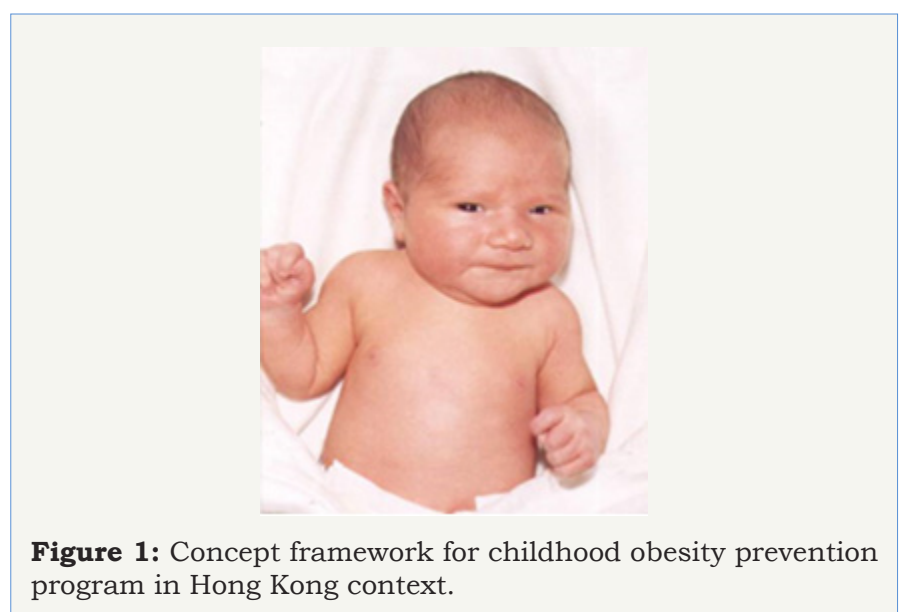

\section{Treatment}

a) Acup: Moxi at Ren8 (Shenque),

b) Needles: Du20(Baihui), Du24 (Shenting), Ren4 (Guanyuan), Ren6 (Qihai),

c) St29 (Guilai), Sp9 (Yinlingquan), Sp6 (Sanyinjiao), Ki 10 (Yingu),

d) Ki3 (Taixi), Ki2(Rangu), Sj5 (Waiguan), Gb41 (Zulinqi),

e) Herbal medicine prescription: Aiye (Artemisia Argyi Levl et Vant)10, Xianmao (Curculigo Orchioides Gaetn.)10, Yinyanghuo (Epimedium Brevicornum Maxim) 15-30, Heshouwu (Polygonum Multiflorum Thunb)10, Danggui (Angelica Sinensis (Oliv.) Diels) 10, Chishao (Paeonia Lactiflora Pall.)10, Tusizi (Cuscuta Chinensis Lam.) 
10, Zishiying (Fluorite) 10, Chuanxiong (Ligusticum Chuanxion Hort.)10, Xiangfu (Cyperus Rotundus L.) 10; Taoren (Prunus Persica (L.) Batsch.) 10, Honghua (Carpesium Tinctorins L.) 10 are added around of ovulation, Chaihu10, Wuyao10 are added before period. A minor variation is made in each week according to her general condition.

\section{Explanation and Discussion}

"(Aiye, (Artemisia Argyi Levl et Vant) Xianmao (Curculigo Orchioides Gaetn.) and Yinyanghuo (Epimedium Brevicornum Maxim) and Tusizi) (Cuscuta Chinensis Lam.)" warm and strengthen kidney“(Yang, Dangui (Angelica Sinensis (Oliv.) Diels) and Heshouwu (Polygonum Multiflorum Thunb))" nourish kidney Yin; "(Chishao (Paeonia Lactiflora Pall.) and Chuanxiong (Ligusticum Chuanxion Hort.))" remove the blood stasis; "(Zishiying (Fluorite))" promote the ovulation; "(Xiangfu (Cyperus Rotundus L.))" pushes the stagnated liver" $(\mathrm{Qi}$; )"moving through."(Taoren (Prunus Persica (L.) Batsch.) and Honghua (Carpesium Tinctorins L.))" are added in the secondary week to emphasize the blood circulation to promote egg's ejaculation, but stop to use after the third week; "(Chaihu (Bupleurum Chinensis DC.) and Wuyao (Lindera Aggregata (Sims) Kostem.))" are added in last week to emphasize removing and warm liver "(Qi)."

Her oestrogen level has increased to 165 after three months and she has her period every other month since. Until being pregnant, she has been accepted TCM for eleven months. A health baby girl has been laboured on term and given eight months of breast-feeding. She starts her regular period, and then she has another pregnancy in a natural way after 18 months of her labour [3].

\section{Results}

This is the case with the early ovaries failure that belongs to an organic diseaseamong female of the reproductive cohorts, so the treatment with only acupuncture at initial, although with patent herbal medicine after are too weak to target her problem. But due to higher efficacy from acupuncture and patent herbal medicine are gained, the patient starts to have some confident to accept TCM treatment, so she is given a regular acupuncture and prescribed the herbal decoction form following her menstrual circle which is needed to give a stronger enough treatment until her menstrual circle recovery and got a successful pregnancy. After a normal pregnancy when she has been preceded a hormonal rebalance, she has been cured from her early failure of ovaries. So she has a natural pregnancy without any support treatment after she breasts feeding and gives a natural birth in her secondary labour.

\section{Conclusion}

Traditional medicine may be better option for nonresponsive reproductive cases on the normal pregnancy.

\section{References}

1. Jiang D (2014) Treating Female Infertility by Strengthening the Natural Menstrual Cycle with Acupuncture and Chinese Herbal Medicine. European Journal of Oriental Medicine 17(6): 28-35.

2. Jin XT, Ma K, Shan J (2014) Clinical efficacy observation on therapy ovulation failure infertility caused by PCOS with reinforcing kidney, activating blood circulation and ovarian stimulation compound recipe. Zhongguo Zhong Yao Za Zhi 39(1): 140-143.

3. Fu Y, Zhao Z, Wu Y, Wu K, Xu X, et al. (2012) Therapeutic mechanisms of Tongmai Dasheng Tablet on tripterygium glycosides induced rat model for premature ovarian failure. J Ethnopharmacol 139(1): 26-33. 